\title{
Doppler beaming factors for white dwarfs, main sequence stars, and giant stars
}

\section{Limb-darkening coefficients for 3D (DA and DB) white dwarf models ${ }^{\star}$}

\author{
A. Claret ${ }^{1,2}$, E. Cukanovaite ${ }^{3}$, K. Burdge ${ }^{4}$, P.-E. Tremblay ${ }^{3}$, S. Parsons ${ }^{5}$, and T. R. Marsh ${ }^{3}$ \\ 1 Instituto de Astrofísica de Andalucía, CSIC, Apartado 3004, 18080 Granada, Spain \\ e-mail: claret@iaa.es \\ 2 Dept. Física Teórica y del Cosmos, Universidad de Granada, Campus de Fuentenueva s/n, 10871 Granada, Spain \\ 3 Department of Physics, University of Warwick, Coventry CV4 7AL, UK \\ ${ }^{4}$ Division of Physics, Mathematics and Astronomy, California Institute of Technology, Pasadena, CA 91125, USA \\ 5 Department of Physics and Astronomy, University of Sheffield, Sheffield S3 7RH, UK
}

Received 18 May 2020 / Accepted 27 July 2020

\begin{abstract}
Context. Systematic theoretical calculations of Doppler beaming factors are scarce in the literature, particularly in the case of white dwarfs. Additionally, there are no specific calculations for the limb-darkening coefficients of 3D white dwarf models.

Aims. The objective of this research is to provide the astronomical community with Doppler beaming calculations for a wide range of effective temperatures, local gravities, and hydrogen/metal content for white dwarfs as well as stars on both the main sequence and the giant branch. In addition, we present the theoretical calculations of the limb-darkening coefficients for 3D white dwarf models for the first time.

Methods. We computed Doppler beaming factors for DA, DB, and DBA white dwarf models, as well as for main sequence and giant stars covering the transmission curves of the Sloan, UBVRI, HiPERCAM, Kepler, TESS, and Gaia photometric systems. The calculations of the limb-darkening coefficients for 3D models were carried out using the least-squares method for these photometric systems.

Results. The input physics of the white dwarf models for which we have computed the Doppler beaming factors are: chemical compositions $\log [\mathrm{H} / \mathrm{He}]=-10.0(\mathrm{DB}),-2.0(\mathrm{DBA})$, and $\mathrm{He} / \mathrm{H}=0$ (DA), with $\log g$ varying between 5.0 and 9.5 and effective temperatures in the range 3750-100 $000 \mathrm{~K}$. The beaming factors were also calculated assuming non-local thermodynamic equilibrium for the case of DA white dwarfs with $T_{\text {eff }}>40000 \mathrm{~K}$. For the mixing-length parameters, we adopted ML2/ $\alpha=0.8$ (DA case) and 1.25 (DB and DBA). The Doppler beaming factors for main sequence and giant stars were computed using the ATLAS9 version, characterized by metallicities ranging from $[-2.5,0.2]$ solar abundances, with $\log g$ varying between 0 and 5.0 and effective temperatures between 3500 and $50000 \mathrm{~K}$. The adopted microturbulent velocity for these models was $2.0 \mathrm{~km} \mathrm{~s}^{-1}$. The limb-darkening coefficients were computed for three-dimensional DA and DB white dwarf models calculated with the $\mathrm{CO}^{5} \mathrm{BOLD}$ radiation-hydrodynamics code. The parameter range covered by the three-dimensional DA models spans $\log g$ values between 7.0 and 9.0 and $T_{\text {eff }}$ between 6000 and $15000 \mathrm{~K}$, while $\mathrm{He} / \mathrm{H}=0$. The three-dimensional DB models cover a similar parameter range of $\log g$ between 7.5 and 9.0 and $T_{\text {eff }}$ between 12000 and $34000 \mathrm{~K}$, while $\log \mathrm{H} / \mathrm{He}=-10.0$. We adopted six laws for the computation of the limb-darkening coefficients: linear, quadratic, square root, logarithmic, power-2, and a general one with four coefficients.

Conclusions. The beaming factor calculations, which use realistic models of stellar atmospheres, show that the black body approximation is not accurate, particularly for the filters $u, u^{\prime}, U, g, g^{\prime}$, and $B$. The black body approach is only valid for high effective temperatures and/or long effective wavelengths. Therefore, for more accurate analyses of light curves, we recommend the use of the beaming factors presented in this paper. Concerning limb-darkening, the distribution of specific intensities for 3D models indicates that, in general, these models are less bright toward the limb than their 1D counterparts, which implies steeper profiles. To describe these intensities better, we recommend the use of the four-term law (also for 1D models) given the level of precision that is being achieved with Earth-based instruments and space missions such as Kepler and TESS (and PLATO in the future).
\end{abstract}

Key words. white dwarfs - binaries: eclipsing - stars: atmospheres

\section{Introduction}

Some time ago, Loeb \& Gaudi (2003) indicated the possibility of detecting exoplanets by using the Doppler beaming technique, which causes an asymmetry in the ellipsoidal modulation in the light curves of binary systems. This technique has an additional

\footnotetext{
* 34 Tables listed in Tables A.1 and A.2 are only available at the CDS via anonymous ftp to cdsarc.u-strasbg. fr (130.79.128.5) or via http://cdsarc.u-strasbg.fr/viz-bin/cat/J/A+A/641/A157
}

advantage because, unlike the transit method, it can be applied to practically any orbital inclination of the system.

The contribution of beaming is essential to synthesizing the light curves of a given system under study. However, such calculations are sparse in the literature. Bloemen (2015) has made an important effort in this regard, but his calculations are limited to a few systems. One of the main objectives of this short paper is to provide users with the calculations of the Doppler beaming factors for white dwarfs, stars on the main sequence, and stars on 
the giant branch. Such calculations cover a wide range of effective temperatures, $\log g$, and hydrogen/metal content, and they are available for some of the most commonly used photometric systems: Sloan, UBVRI, Kepler, TESS, Gaia, and HiPERCAM.

On the other hand, some studies have shown that 3D atmosphere models compare better with observations of the center to limb variation for the Sun (see Pereira et al. 2013). Pereira et al. (2013) find a very good agreement between the limbdarkening from 3D models and observations at ultraviolet and visible wavelengths. The observations used in their paper were those of Pierce \& Slaughter (1977) and Neckel \& Labs (1994). However, Pereira et al. (2013) also find a poorer agreement for longer wavelengths (between 12000 and $24000 \AA$ ). In order to test the validity of stellar atmosphere models in other kind of stars, we present a series of computations of limb-darkening coefficients (LDCs) for white dwarfs of spectral types DA and $\mathrm{DB}$, based on 3D model atmospheres and covering the same range of photometric systems as mentioned above.

We organize the paper as follows: Section 2 is dedicated to the computation of the Doppler beaming factors while Sect. 3 is devoted to the LDCs of DA-3D and DB-3D white dwarf models. Finally, in Appendix A we give brief explanations of Tables A.1 and A.2 and the data they contain. Additional material or calculations for specific photometric systems can be carried out upon request.

\section{Doppler beaming factors for DA, DB, and DBA white dwarfs, main sequence stars, and giant stars}

Doppler beaming is caused by the radial velocity of the stars in a double system, which displace the spectrum and modulate the photon emission rate toward the observer. The first theoretical investigation into this effect was carried out by Hills \& Dale (1974) in the case of rotating white dwarfs and by Shakura \& Postnov (1987) for the orbital movement of double systems.

From the observational point of view, there have also been important advances related to beaming effects. We can mention, for example: Mazeh \& Faigler (2010), who detected an ellipsoidal modulation and relativistic beaming effect in the exoplanetary system CoRot-3; and Ehrenreich et al. (2011), who, using spectroscopic techniques, confirmed the relativistic beaming effect in the KOI-74 system consisting of a hot compact object around an early-type star. More recently, Wong et al. (2020) performed a consistent analysis of the KOI-964 system, which consists of a hot white dwarf and an A-type host star. With respect to the Doppler beaming factor, Wong et al. (2020) find a good agreement between theory and observational data.

In a previous paper on gravity and LDCs (Claret et al. 2020), we computed the beaming factors for four white dwarf models - DA, DB, DBA, and non-local thermodynamic equilibrium (NLTE) DA - adopting the black body approach. However, such a simple method neglects the effects of the Balmer jump, of the absorption lines, and even of the shape of the spectra, which is different from black body radiation. Furthermore, such an approach does not predict any dependence on $\log g$ or atmospheric composition. As we will see below, the local gravity and the hydrogen content (or metallicity in the case of stars) may influence the calculation of the beaming factors.

As mentioned in the Introduction, there are no systematic calculations for beaming factors that consider realistic atmosphere models of white dwarfs or stars on the main sequence or the giant branch. In the present paper, we adopt white dwarf atmosphere models of the types DA, DB, DBA, and DANLTE as well as the ATLAS9 models (main sequence, subgiants, and giants) for the calculation of the beaming factors for several passbands. The description of the adopted white dwarf models (DA, DB, DBA, and DA-NLTE) can be found in Claret et al. (2020, Sect. 2). The models for stars on the main sequence, subgiants, and giants used here were calculated with the ATLAS9 version. The computation of beaming factors are presented for metallicities ranging from $[-2.5,0.2]$ solar abundances, with $\log g$ varying between 0 and 5.0 and effective temperatures between $3500 \mathrm{~K}$ and $50000 \mathrm{~K}$. The adopted microturbulent velocity is $2.0 \mathrm{~km} \mathrm{~s}^{-1}$. For more detailed information on ATLAS9 features, see, for example, Castelli et al. (1997).

If we assume that the monochromatic flux is proportional to a frequency power, that is to say $F(v) \propto v^{\alpha}$ (Shakura \& Postnov 1987), for the flux variation due to the Doppler shift (Loeb \& Gaudi 2003) we have

$F(v)_{\mathrm{obs}}=F(v)_{0}\left(1+(3-\alpha) \frac{v_{r}}{c}\right)$,

where $v_{r}$ is the radial velocity (nonrelativistic approach), $c$ is the velocity of light in vacuum, $F(v)_{\text {obs }}$ is the observed flux, and $F(v)_{0}$ is the monochromatic flux in the absence of the source motion. Eq. (1) can also be written as

$F(\lambda)_{\mathrm{obs}}=F(\lambda)_{0}\left(1-B(\lambda) \frac{v_{r}}{c}\right)$,

where $\lambda$ is the wavelength and $B(\lambda)$ is the monochromatic beaming factor,

$B(\lambda)=5+\frac{d \ln F(\lambda)}{d \ln \lambda}$.

To compute the photon weighted bandpass-integrated beaming factors, we used the following equation:

$\bar{B}=\frac{\int_{\lambda 1}^{\lambda 2} S(\lambda) \lambda B(\lambda) F(\lambda) \mathrm{d} \lambda}{\int_{\lambda 1}^{\lambda 2} S(\lambda) \lambda F(\lambda) \mathrm{d} \lambda}$,

where $S(\lambda)$ is the response function for each passband. In order to generate the average beaming factors, we adopted in Eq. (4) the transmission curves for the Sloan $\left(u^{\prime} g^{\prime} r^{\prime} i^{\prime} z^{\prime} y^{\prime}\right), U B V R I$, HiPERCAM, Kepler, TESS, and Gaia passbands. We did not consider reddening in the present calculations. We note that Eq. (4) is suitable for photon-counting devices, such as CCDs.

Figure 1 shows the comparison between beaming factors computed using Eq. (4) (continuous line) and computed adopting the black body approach (stars) for HiPERCAM and using DA models at $\log g=5.0$. The differences between the two recipes are notable, particularly for shorter effective wavelengths. Also noteworthy is the peak of beaming in the near-ultraviolet region around $T_{\text {eff }}=10000 \mathrm{~K}$. This feature is also detected for less compact star models generated with the ATLAS9 code (see Fig. 2). Such behavior is related to the strength of the Balmer jump, whose effect is more intense for models with $T_{\text {eff }} \approx 10000 \mathrm{~K}$. On the other hand, if we assume that the flux is given by the Planck function, then for large values of $\lambda$ and/or $T_{\text {eff }}$ the calculations based on the black body approximation are closer to the predictions given by Eq. (4) using realistic atmosphere models $(\approx 1.0)$, as expected.

Unlike the black body approach, the calculations that adopt more realistic models of stellar atmospheres predict a dependence of the beaming factors on the local gravity, as can be 

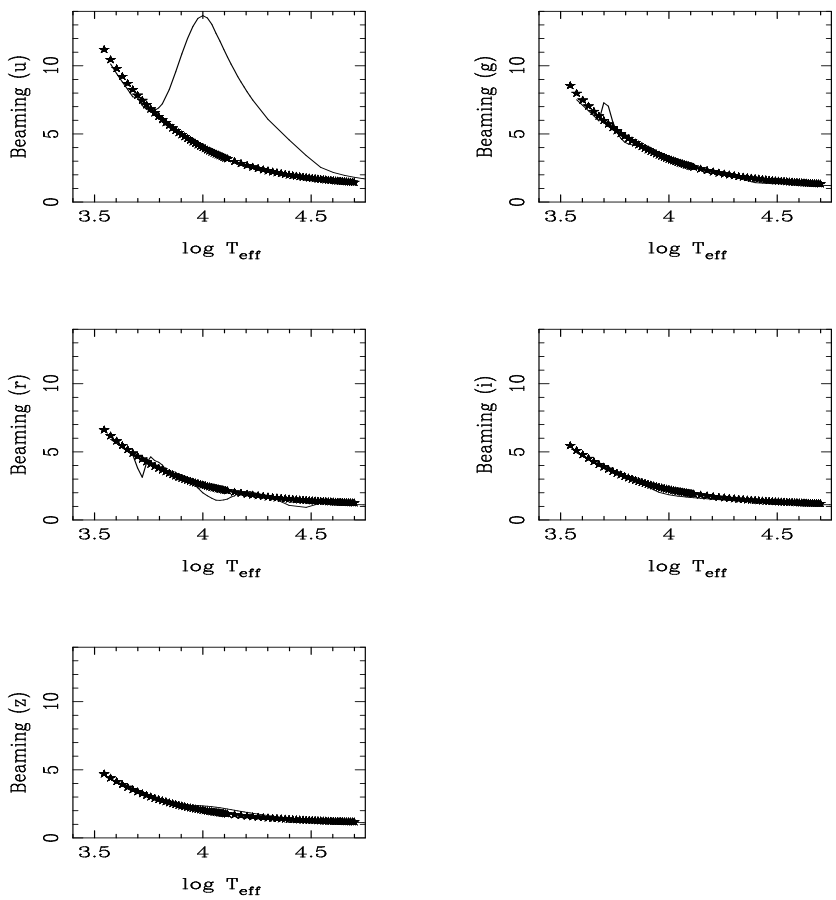

Fig. 1. Comparison between the beaming factors according to Eq. (4) (HiPERCAM passbands). The DA white dwarf models are represented by continuous lines and black body approximation by stars. All models are at $\log g=5.0$.

seen in Fig. 3. The differences in beaming factors due to $\log g$ are more prominent for shorter wavelengths, although for the $y^{\prime}$ passband there is also a notable difference for cooler models. For hot models and long effective wavelengths, the beaming factors are almost independent of $\log g$ because the models are close to the Rayleigh-Jeans law of a black body. The effects of local gravity on the beaming factors are very interesting, particularly for the Sloan $\left(u^{\prime}\right)$ filter. In general, the strength of the Balmer jump decreases with increasing local gravity of the white dwarf model (because of nonideal gas effects), and this impacts the beaming calculations for shorter effective wavelengths. The computation of the beaming factors depends on the strength of the Balmer jump, which in turn depends on the local gravity and effective temperature. We note that the RayleighJeans law and continuum opacities also contribute to the Balmer jump.

In Fig. 4, we show the influence of the hydrogen abundance in the beaming factor profiles. The differences are small for longer effective wavelengths but they can be detected observationally, particularly for effective temperatures below $20000 \mathrm{~K}$ and for shorter effective wavelengths. As we anticipated at the beginning of this section, realistic atmosphere models for white dwarfs are able to predict a beaming factor dependence on the local gravity and with the hydrogen content, particularly for shorter effective wavelengths.

Figure 5 illustrates the differences between the beaming factors for DA and DA-NLTE models for the Sloan passbands. The beaming factors computed for DA models are systematically larger than the corresponding DA-NLTE models for the entire instrumental range and for all considered effective temperatures. It is interesting to compare the systematic differences between the DA and DA-NLTE models with the results shown in Fig. 10 in Claret et al. (2020), where the effects of NLTE on the gravitydarkening coefficients (GDCs) are shown. From that figure, it

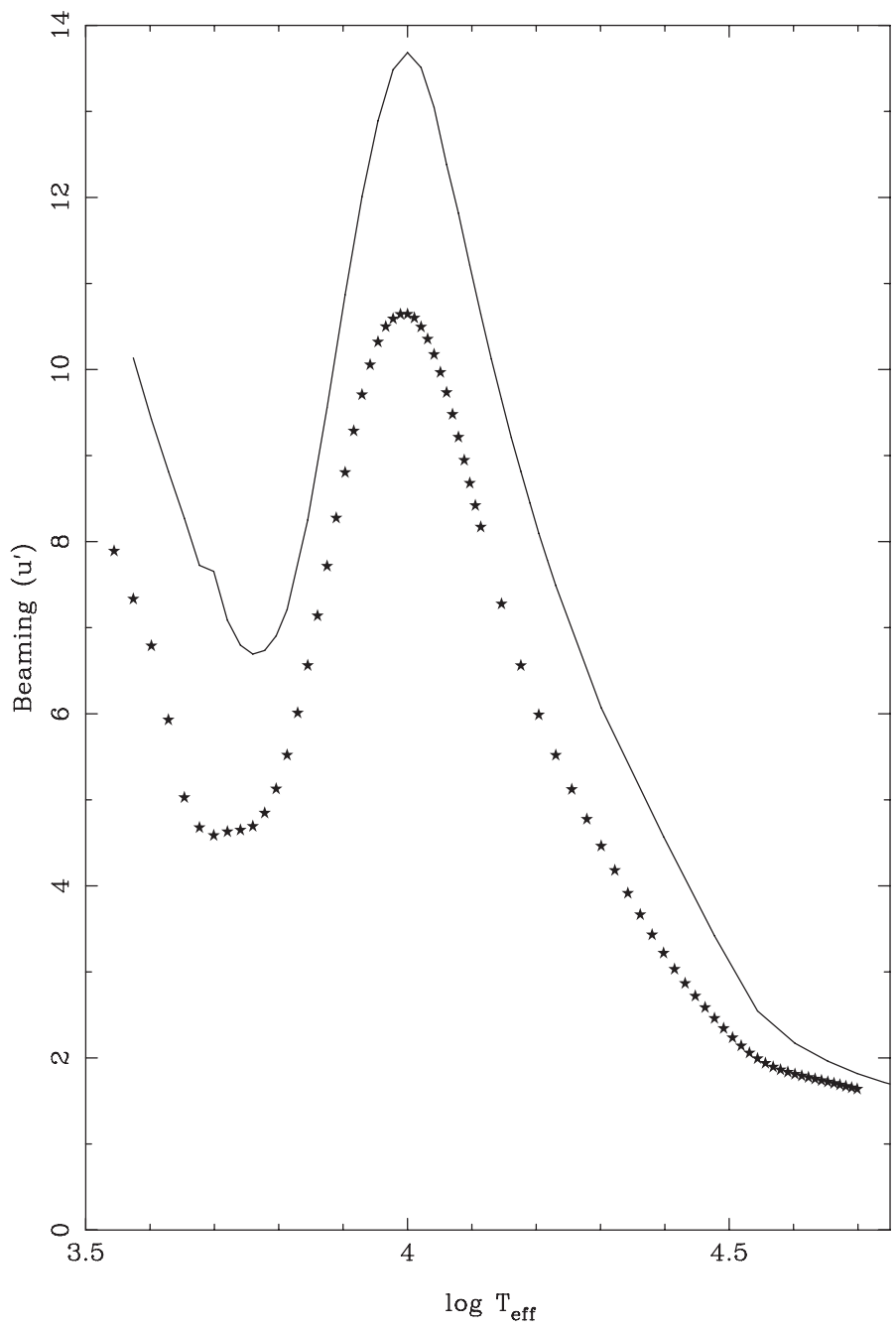

Fig. 2. Same as in Fig. 6 but for the $u^{\prime}$ passband.

was possible to determine the effective temperature limit for which it is necessary to adopt NLTE, around $40000 \mathrm{~K}$, in agreement with the results by Gianninas et al. (2013), although this indicator cannot be considered as definitive. The "inefficiency" of the beaming factors in distinguishing this effective temperature limit may be due to the fact that, in the case of GDC calculations, we consider derivatives of central specific intensities with respect to Teff and $\log g$ (whose values are relatively small) while calculating the beaming factors the derivatives $\left(\frac{\mathrm{d} \ln F(\lambda)}{\mathrm{d} \ln \lambda}\right)$ are very much larger, especially at the Balmer jump and in the environment of spectral lines. Another complementary possibility to explain the appearance of Fig. 5 is likely related to the use of different codes for LTE and NLTE.

Concerning the ATLAS9 models, Figure 6 shows the beaming factors for the Kepler, TESS, and Gaia passbands for models on the main sequence (solar metallicity, stars) and DA white dwarf models, denoted by continuous lines for a fixed value of $\log g=5$.0. In general, the differences are not very large because we are dealing with relatively long wavelengths. The situation changes for the ultraviolet range. In Fig. 2, we show the behavior of beaming factors of the same models as in Fig. 6 but for the $u^{\prime}$ filter; the differences are notable in magnitude, in addition to being systematically offset. The systematic effects illustrated in Fig. 2 are due to the fact that the respective codes adopt different equations of state and helium content. 

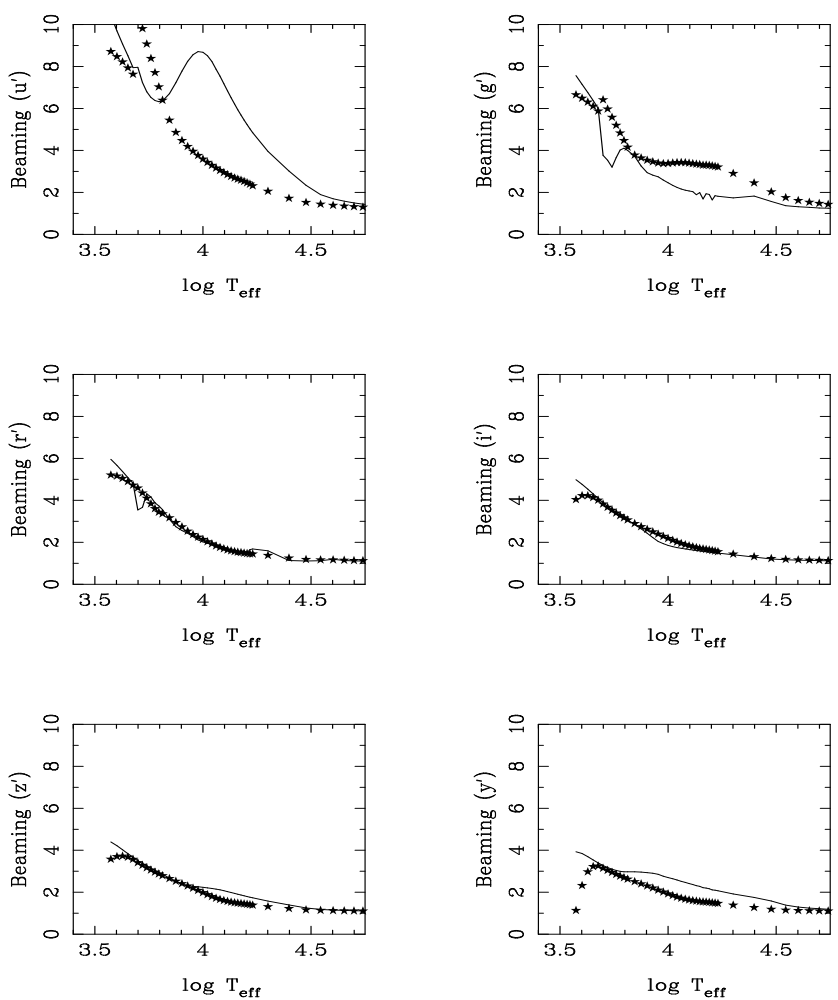

Fig. 3. Comparison between the beaming factors (Sloan passbands, DA white dwarf models) according to Eq. (4). Continuous lines indicate models with $\log g=5.0$ while stars denote those with $\log g=9.0$.

The comparison between theoretical and semi-empirical beaming factors is a difficult task mainly due to the scarcity of observational data. Here we analyze the semi-empirical beaming data related to white dwarfs in the passband $g^{\prime}$, published by Shporer et al. (2010). These authors, analyzing the system NLTT 11748, a non-interacting eclipsing double white dwarf binary with optical light dominated by an extremely low mass (ELM) DA white dwarf, determined $\overline{B\left(g^{\prime}\right)}=3.0 \pm 0.4$. The system consists of He-core and $\mathrm{C} / \mathrm{O}$-core white dwarfs but both atmospheres are pure hydrogen. We present in Fig. 7 an exploratory comparison between the theoretical predictions for the passband $g^{\prime}$ and the value inferred in Shporer et al. (2010). The theoretical beaming compares well with the derived semiempirical value. On the other hand, Bloemen et al. (2011), studying the KPD $1946+4340$ system, observationally determined $\overline{B(\text { Kepler })}=1.33 \pm 0.02$ for the sdB star. Since we did not calculate the beaming factors specifically for sdB-type stars, we used the results of DA and DB models to compare. Although this comparison is not entirely consistent, it can provide us with some clues. The respective values of the beaming factors are 1.34 and 1.32 and are in agreement with the semi-empirical value given by Bloemen et al. (2011).

The beaming factor calculations for stars on the main sequence or the giant branch are also scarce in the literature. Bloemen et al. (2012), for example, present the calculation of the beaming factor for an A-type main-sequence star (KOI-74) in the Kepler passband and find $\bar{B}=2.22 \pm 0.04$. An inspection in our Table 9 (see Table A.1) shows that the value of the beaming factor for the ATLAS9 model $\left(T_{\text {eff }}=9500 \mathrm{~K}, \log g=4.3\right.$, and solar metallicity) is around 2.21 , in good agreement with both the value mentioned above and the semi-empirical value $(2.24 \pm 0.05)$.
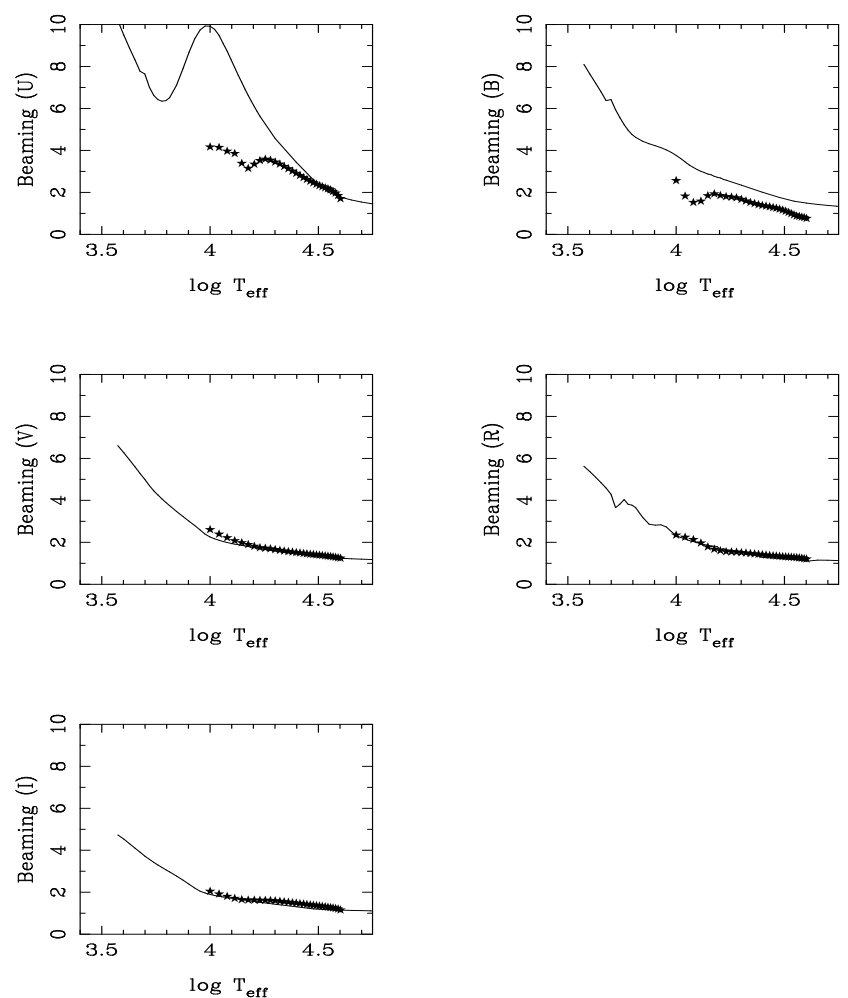

Fig. 4. Beaming factors for the UBVRI passbands according to Eq. (4) for $\log g=5.0$. Continuous lines indicate DA white dwarf models while stars denote DB models.

\section{Limb-darkening coefficients for DA-3D and DB-3D models}

Claret et al. (2020) derived LDCs for a set of one-dimensional DA, DB, and DBA white dwarf models. Here we compare these results to $3 \mathrm{D}$ model atmospheres for DA white dwarfs (DA3D; Tremblay et al. 2013) in the ranges $\log g=7.0-9.0$ and $T_{\text {eff }}=6000-15000 \mathrm{~K}$, and for DB/DBA white dwarfs (DBA3D; Cukanovaite 2018, 2019 in the ranges $\log g=7.5-9.0$ and $T_{\text {eff }}=12000-34000 \mathrm{~K}$. As usual, we adopted the following limb-darkening laws for the DA-3D and DB-3D models: the linear law (Schwarzschild 1906; Russell 1912; Milne 1921)

$\frac{I(\mu)}{I(1)}=1-u(1-\mu)$

the quadratic law (Kopal 1950)

$\frac{I(\mu)}{I(1)}=1-a(1-\mu)-b(1-\mu)^{2}$,

the square-root law (Díaz-Cordovés \& Giménez 1992)

$\frac{I(\mu)}{I(1)}=1-c(1-\mu)-d(1-\sqrt{\mu})$,

the logarithmic law (Klinglesmith \& Sobieski 1970)

$\frac{I(\mu)}{I(1)}=1-e(1-\mu)-f \mu \ln (\mu)$,

the power-2 law (Hestroffer 1997)

$\frac{I(\mu)}{I(1)}=1-g\left(1-\mu^{h}\right)$, 

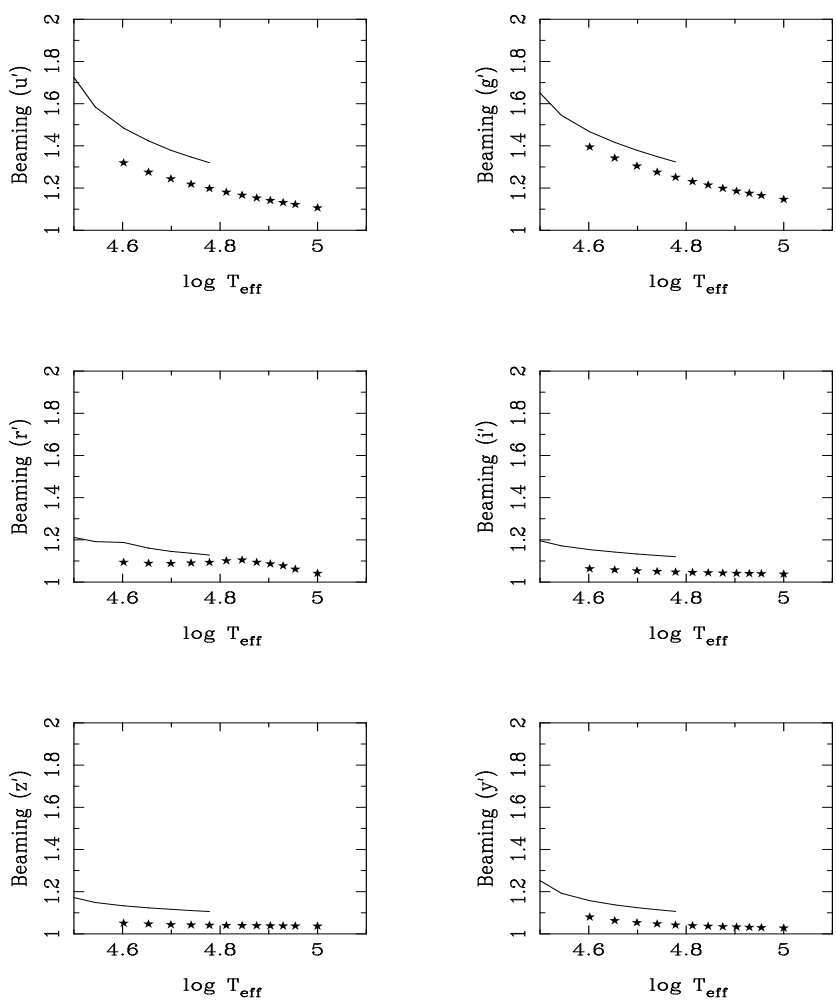

Fig. 5. Beaming factors (Sloan passbands) according to Eq. (4) for $\log g=7.0$. Continuous lines indicate LTE DA models and stars indicate NLTE DA models.
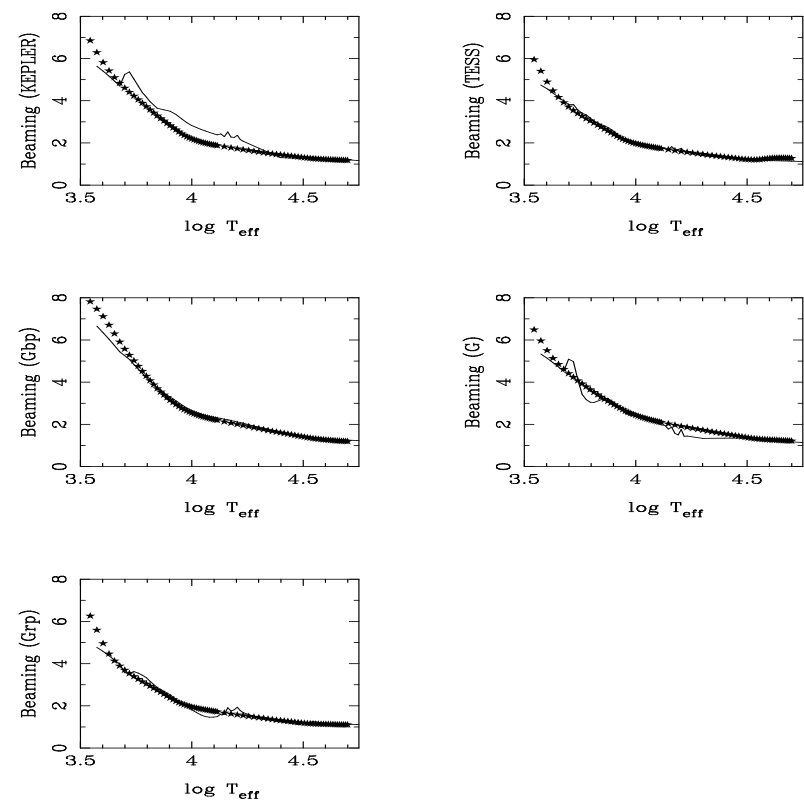

Fig. 6. Beaming factors for main sequence ATLAS9 models (solar metallicity, stars) and DA models (continuous lines) according to Eq. (4). All models are at $\log g=5.0$ and shown for the Kepler, TESS and Gaia Gbp, G, and Grp passbands.

and a four-term law (Claret 2000)

$\frac{I(\mu)}{I(1)}=1-\sum_{k=1}^{4} a_{k}\left(1-\mu^{\frac{k}{2}}\right)$,

where $I(1)$ is the specific intensity at the center of the disk and $u, a, b, c, d, e, f, g, h$, and $a_{k}$ are the corresponding LDCs. For the

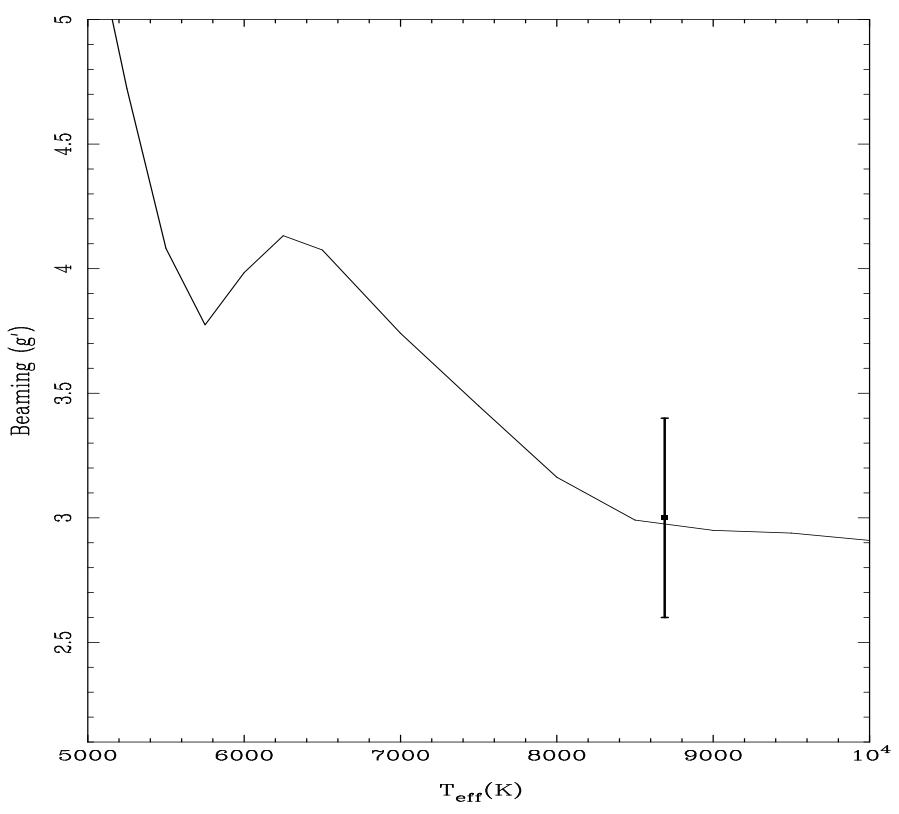

Fig. 7. Comparison between the semi-empirical beaming factor (error bars) for the system NLTT 11748 for the $g^{\prime}$ passband and theoretical predictions adopting DA models at $\log g=6.5$.

calculation of the LDCs, we adopted the least-squares method (LSM). For details regarding this choice, see Claret et al. (2020).

The merit function for each law and passband is given by

$\chi^{2}=\sum_{i=1}^{N}\left(y_{i}-Y_{i}\right)^{2}$,

where $y_{i}$ is the model intensity at point $i, Y_{i}$ is the fitted function at the same point, and $N$ is the number of $\mu$ points. The $\chi^{2}$ are given in Tables 11-34 (see Appendix A) for user orientation for each law and passband.

The advantages of the four-term law over all bi-parametric or linear laws are widely discussed in Claret (2000) for main sequence and giant stars, and for the case of white dwarfs in Claret et al. (2020). In the case of 3D models for main sequence stars and giants, a discussion on the superiority of the four-term law can be found in Magic et al. (2015). In the case of the DA-3D and DB-3D models, the corresponding difference in $\chi^{2}$ between the six laws gives similar results to those obtained in the aforementioned papers.

In our previous paper on LDCs and GDCs for white dwarfs, we only investigated 1D models. Our main objective in this section is to study the effects of 3D models on LDCs and GDCs. However, for the calculation of the GDCs, we need to calculate the term $\left(\frac{\partial \ln I_{o}(\lambda)}{\partial \ln g}\right)_{T_{\text {eff }}}$, where $I_{o}(\lambda)$ is the specific intensity at a given wavelength at the center of the stellar disk and the subscript $T_{\text {eff }}$ denotes a derivative at constant effective temperature. The impact of this contribution to the GDC is not very large, but it is not negligible. Unfortunately, the present 3D grids are not regular with respect to effective temperatures, which prevents us from performing consistent calculations of GDCs. We will address these calculations in the future, since they require an enormous amount of personal and computational time. However, we can calculate the GDCs for an individual system upon request from interested parties.

Before analyzing the LDCs for the white dwarfs, we will note that a very important but sometimes overlooked point is that a close binary system should not be treated as if each star in 

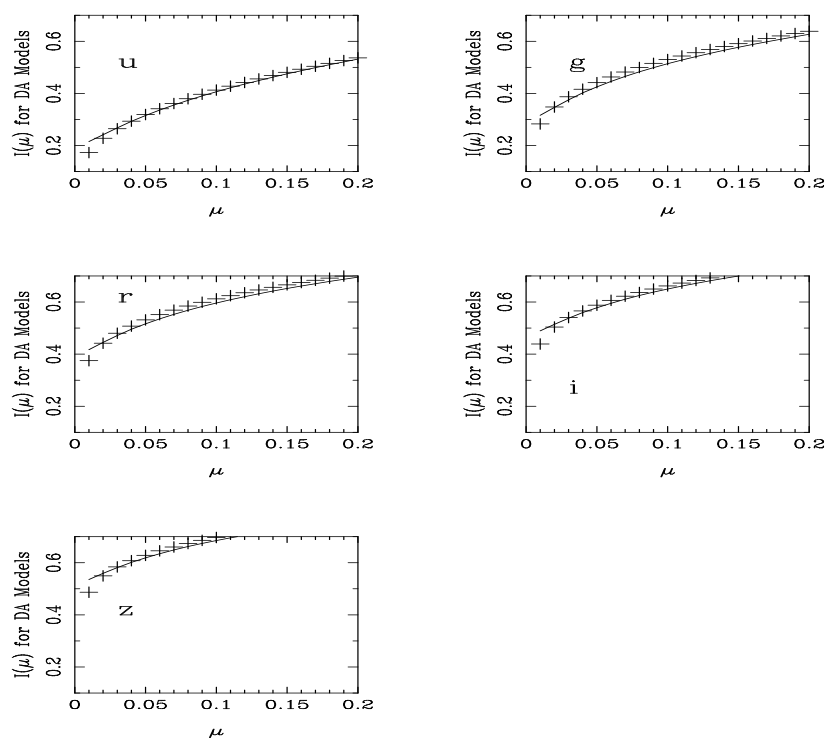

Fig. 8. Distribution of specific intensities for 1D (continuous line) and 3D (crosses) DA models with $T_{\text {eff }}=6000 \mathrm{~K}$ and $\log g=8.0$ for the HiPERCAM instrumental system.
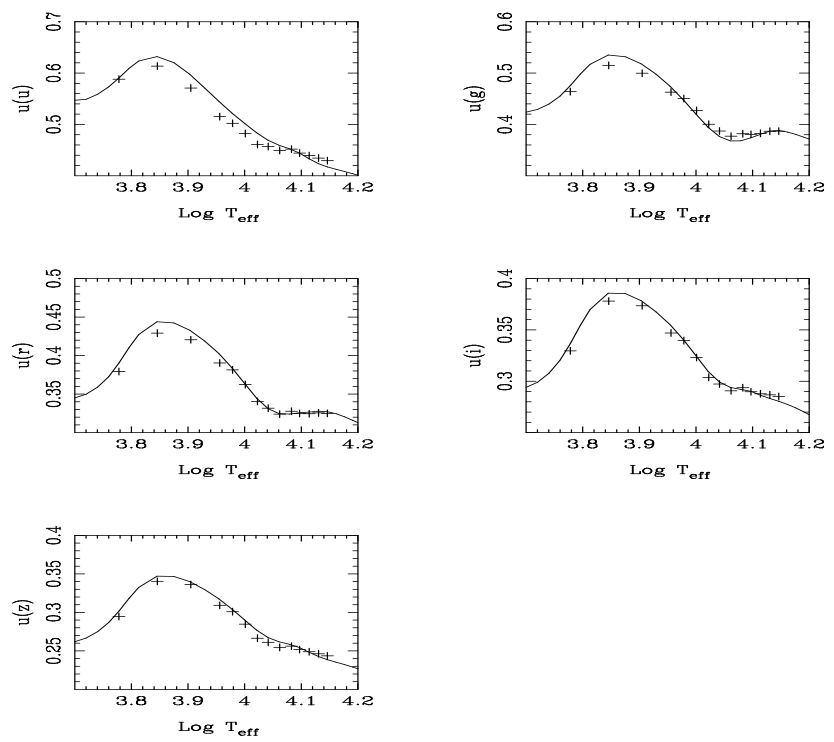

Fig. 9. Comparison between linear LDCs for HiPERCAM passbands for DA-3D models (crosses) and DA-1D models (continuous line) at $\log g=7.0$.

the pair behaves as an isolated star, since the proximity effects can change the individual properties of each star. For example, mutual irradiation between the components alters the spectra, occasionally resulting in a very different spectrum from a nonirradiated white dwarf. This can alter the distribution of specific intensities (limb-darkening), effective temperatures, and even the observed chemical compositions. In many cases, this type of interaction can lead to erroneous determinations of the stellar parameters as indicated by Claret \& Giménez $(1990,1992)$ and Claret $(2001,2004,2007)$. In this section, we work with the hypothesis that the stars in a close binary system behave like isolated stars, but we plan to implement the proximity effects in the future.

In Fig. 8, we compare the behavior of the specific intensities as a function of $\mu$ for the one- and three-dimensional DA models with $T_{\text {eff }}=6000 \mathrm{~K}$ and $\log g=8.0$ (HiPERCAM). We note that
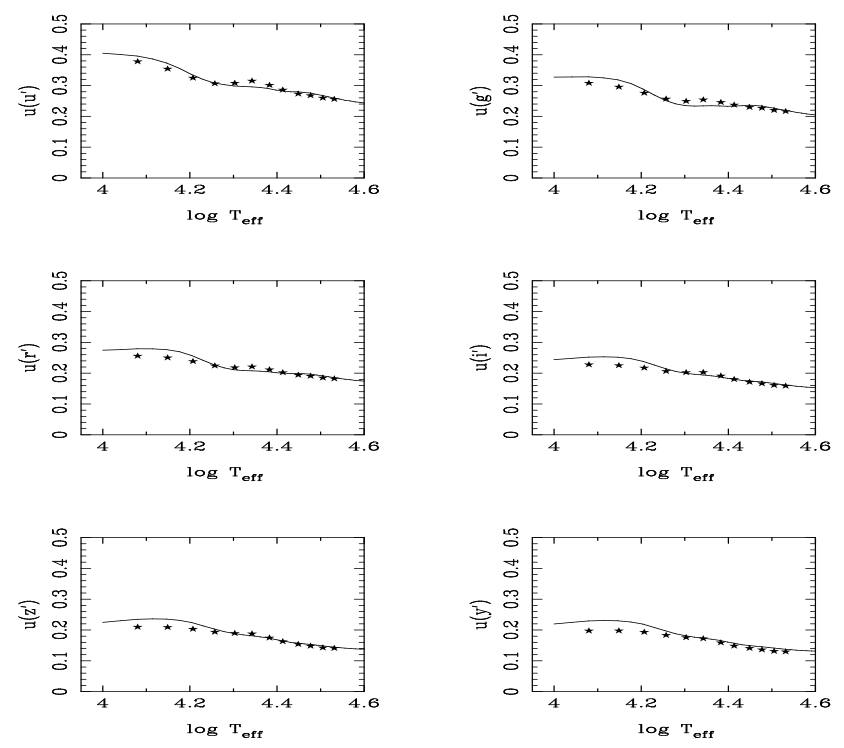

Fig. 10. Comparison between linear LDCs and Sloan passbands for DB3D models (stars) and DB-1D models (continuous line) at $\log g=8.0$.

the effective temperature is not exactly the same as the $T_{\text {eff }}$ of the 3D model, which is $5998 \mathrm{~K}$. However, this difference is essentially negligible for comparison purposes. Examining Fig. 8, we can see a general trend that the $1 \mathrm{D}$ model is brighter than the corresponding 3D model toward the limb for all passbands of the HiPERCAM instrumental system. Such an effect has been previously noted by Magic et al. (2015) when these authors compared the distribution of intensities for the ATLAS (1D) and Stagger (3D) models of main sequence stars. We also find that this effect depends on the local gravity: the larger the $\log g$, the smaller the effect, provided the other parameters of the input physics are fixed. An explanation for this effect may be that the temperature stratification and temperature gradients are different in the 1D and 3D modeling Magic et al. (2015). One contribution that changes the temperature stratification for DA and DB white dwarfs is the convective overshoot seen in 3D models, which cools the upper layers (Tremblay et al. 2013; Cukanovaite 2018). Since regions toward the limb have a longer optical path across the overshoot region, they become less bright in 3D.

On the other hand, as is widely known, the linear approximation for limb-darkening does not correctly describe the distribution of specific intensities. However, this law allows us to more easily compare the results from models with different input physics. We will use it here for the purpose of studying the direct effects of 3D on LDCs. Comparisons of the LDCs between DA-1D and DA-3D models are shown in Fig. 9 (HiPERCAM). Significant differences can only be distinguished for shorter effective wavelengths and lower effective temperatures, and these could be at the limit of observational detection. Three-dimensional effects due to convective energy transport become significant at low $T_{\text {eff }}$ and thus the differences between $1 \mathrm{D}$ and $3 \mathrm{D}$ at these temperatures are not unexpected.

In Fig. 10, we compare the linear LDCs for DB-3D models (stars) with their 1D counterparts. There is a systematic effect in the sense that the LDCs of the 3D models are smaller for $T_{\text {eff }}<$ 20000 K. Similarly to DA white dwarfs, 3D effects become significant in this temperature regime (Cukanovaite 2018).

According to the results shown in Fig. 8, the distribution of specific intensities for 3D models indicates that, in general, these 
models are less bright than their 1D counterparts, which implies steeper profiles toward the limb. To better describe these intensities, we recommend the use of the four-term law given the level of precision that is being achieved with Earth-based instruments as well as space missions, such as Kepler, TESS, and, in the near future, PLATO.

\section{Summary}

The beaming factor calculations presented here, based on more realistic atmosphere models, clearly indicate that the black body approximation is not accurate. This is particularly important for the filters $u, u^{\prime}, U, g, g^{\prime}$, and $B$ since the black body approach is only valid for high effective temperatures and/or long effective wavelengths. To check the validity of our calculations of beaming factors, we compared them with the few existing cases in the literature (theoretical and semi-empirical), finding a very good agreement. To more accurately analyze the light curves of white dwarfs, we recommend the use of the beaming factors presented here. On the other hand, concerning limb-darkening, we recommend the use of the 3D four-term law since Hayek et al. (2012) has shown that 3D models lead to a better fit for the HD 189733 system when compared to the predictions of the 1D models. Finally, Tables A.1 and A.2 give information on the data available at the CDS (Centre de Données Astronomiques de Strasbourg) or directly from the authors.

Acknowledgements. We thank the referee S. Bloemen for his helpful suggestions and comments. The Spanish MEC (ESP2017-87676-C5-2-R, PID2019107061GB-C64, and PID2019-109522GB-C52) is gratefully acknowledged for its support during the development of this work. A. C. also acknowledges financial support from the State Agency for Research of the Spanish MCIU through the "Center of Excellence Severo Ochoa" award for the Instituto de Astrofísica de Andalucía (SEV-2017-0709). The research leading to these results has received funding from the European Research Council under the European Union's Horizon 2020 research and innovation programme n. 677706 (WD3D). SGP acknowledges the support of a Science and Technology Facilities Council (STFC) Ernest Rutherford Fellowship. This research has made use of the SIMBAD database, operated at the CDS, Strasbourg, France, and of NASA's Astrophysics Data System Abstract Service.

\section{References}

Bloemen, S. 2015, High-Precision Studies of Compact Variable Stars (Springer Theses)

Bloemen, S., Marsh, T. R., Ostensen, R. H., et al. 2011, MNRAS, 410, 1787

Bloemen, S., Marsh, T. R., Degroote, P., et al. 2012, MNRAS, 422, 2600

Castelli, A., Gratton, R. G., \& Kurucz, R. L. 1997, A\&A, 324, 432

Claret, A. 2000, A\&A, 363, 1081

Claret, A. 2001, MNRAS, 327, 989

Claret, A. 2004, A\&A, 422, 66

Claret, A. 2007, A\&A, 470, 1099

Claret, A., \& Giménez, A. 1990, A\&A, 230, 412

Claret, A., \& Giménez, A. 1992, A\&A, 256, 572

Claret, A., Cukanovaite, E., Burdge, K., et al. 2020, A\&A, 634, A93

Cukanovaite, E., et al. 2018, MNRAS, 481, 1522

Cukanovaite, E., et al. 2019, MNRAS, 490, 1010

Díaz-Cordovés, J., \& Giménez, A. 1992, A\&A, 259, 227

Ehrenreich, D., Lagrange, A.-M., Bouchy, F., et al. 2011, A\&A, 525, A85

Gianninas, A., Strickland, B. D., Kilic, M., \& Bergeron, P. 2013, ApJ, 765, 102

Hayek, W., Sing, D., \& Pont, A. M. 2012, A\&A, 539, A102

Hestroffer, D. 1997, A\&A, 327, 199

Hills, J. G., \& Dale, T. M. 1974, A\&A, 30, 135

Klinglesmith, D. A., \& Sobieski, S. 1970, AJ, 75, 175

Kopal, Z. 1950, HarCi, 454, 1

Loeb, A., \& Gaudi, B. S. 2003, ApJ, 588, L117

Magic, Z., Chiavassa, A., Collet, R., \& Asplund, M. 2015, A\&A, 573, A90

Mazeh, T., \& Faigler, S. 2010, A\&A, 521, A59

Milne, E. A. 1921, MNRAS, 81, 361

Neckel, H., \& Labs, D. 1994, SoPh, 153, $91 \mathrm{~N}$

Pereira, T. M. D., Asplund, M., Collet, R., et al. 2013, A\&A, 554, A118

Pierce, A. K., \& Slaughter, C. D. 1977, Soph, 51, 25

Russell, H. N. 1912, ApJ, 36, 54

Shakura, N. I., \& Postnov, A. 1987, A\&A, 183, L21

Schwarzschild, K. 1906, Nachr. Ges. Wiss. Gottingen Math.-Phy. K1., 43

Shporer, A., Kaplan, D. L., Steinfadt, J. D. R., et al. 2010, ApJ, 725, L200

Tremblay, P.-E., Ludwig, H.-G., Steffen, M., \& Freytag, B. 2013, A\&A, 559, A104

Wong, I., Shporer, A., Becker, J. C., et al. 2020, AJ, 159, 29 


\section{Appendix A: Brief description of online data}

Tables A.1 and A.2 summarize the types of data available as well as the central intensities for each photometric system in ergs $\mathrm{cm}^{-2} \mathrm{~s}^{-1} \mathrm{~Hz}^{-1} \mathrm{ster}^{-1}$ in the case of LDCs for the 3D models. For more details, see the ReadMe file at the CDS website.

Table A.1. Doppler beaming factors for the Kepler, TESS, Gaia, HiPERCAM, Sloan, and UBVRI photometric systems.

\begin{tabular}{lccccc}
\hline \hline Name & Source & Range $T_{\text {eff }}$ & Range $\log g$ & $\log [\mathrm{H} / \mathrm{He}]$ & Beaming/Filters \\
\hline Table 1 & DA & $3750 \mathrm{~K}-60000 \mathrm{~K}$ & $5.0-9.5$ & 0.00 & Kepler/TESS/Gaia/HiPERCAM \\
Table 2 & DB & $10000 \mathrm{~K}-40000 \mathrm{~K}$ & $5.5-9.5$ & -10.0 & Kepler/TESS/Gaia/HiPERCAM \\
Table 3 & DBA & $11000 \mathrm{~K}-40000 \mathrm{~K}$ & $7.0-9.0$ & -2.0 & Kepler/TESS/Gaia/HiPERCAM \\
Table 4 & DA-NLTE & $40000 \mathrm{~K}-100000 \mathrm{~K}$ & $6.0-9.5$ & 0.0 & Kepler/TESS/Gaia/HiPERCAM \\
Table 5 & DA & $3750 \mathrm{~K}-60000 \mathrm{~K}$ & $5.0-9.5$ & 0.0 & Sloan/UBVRI \\
Table 6 & DB & $10000 \mathrm{~K}-40000 \mathrm{~K}$ & $5.5-9.5$ & -10.0 & Sloan/UBVRI \\
Table 7 & DBA & $11000 \mathrm{~K}-40000 \mathrm{~K}$ & $7.0-9.0$ & -2.0 & Sloan/UBVRI \\
Table 8 & DA-NLTE & $40000 \mathrm{~K}-100000 \mathrm{~K}$ & $6.0-9.5$ & 0.0 & Sloan/UBVRI \\
Table 9 & ATLAS9 & $3500 \mathrm{~K}-50000 \mathrm{~K}$ & $0.0-5.0$ & $-2.5-0.5^{(a)}$ & Kepler/TESS/Gaia/HiPERCAM \\
Table 10 & ATLAS9 & $3500 \mathrm{~K}-50000 \mathrm{~K}$ & $0.0-5.0$ & $-2.5-0.5^{(a)}$ & Sloan/UBVRI \\
\hline
\end{tabular}

Notes. ${ }^{(a)}$ Values of metallicities for the ATLAS9 models.

Table A.2. Limb-darkening coefficients for the Kepler, TESS, Gaia, HiPERCAM, Sloan, and UBVRI photometric systems.

\begin{tabular}{|c|c|c|c|c|c|c|}
\hline Name & Source & Range $T_{\text {eff }}$ & Range $\log g$ & $\log [\mathrm{H} / \mathrm{He}]$ & Filters & Fit/Equations \\
\hline Table 11 & DA-3D & $5998 \mathrm{~K}-14986 \mathrm{~K}$ & $7.0-9.0$ & 0.0 & Kepler/TESS/Gaia/HiPERCAM & LSM/Eq. (4) \\
\hline Table 12 & DA-3D & $5998 \mathrm{~K}-14986 \mathrm{~K}$ & $7.0-9.0$ & 0.0 & Kepler/TESS/Gaia/HiPERCAM & LSM/Eq. (5) \\
\hline Table 13 & DA-3D & $5998 \mathrm{~K}-14986 \mathrm{~K}$ & $7.0-9.0$ & 0.0 & Kepler/TESS/Gaia/HiPERCAM & LSM/Eq. (6) \\
\hline Table 14 & DA-3D & $5998 \mathrm{~K}-14986 \mathrm{~K}$ & $7.0-9.0$ & 0.0 & Kepler/TESS/Gaia/HiPERCAM & LSM/Eq. (7) \\
\hline Table 15 & DA-3D & $5998 \mathrm{~K}-14986 \mathrm{~K}$ & $7.0-9.0$ & 0.0 & Kepler/TESS/Gaia/HiPERCAM & LSM/Eq. (8) \\
\hline Table 16 & DA-3D & $5998 \mathrm{~K}-14986 \mathrm{~K}$ & $7.0-9.0$ & 0.0 & Kepler/TESS/Gaia/HiPERCAM & LSM/Eq. (9) \\
\hline Table 17 & DA-3D & $5998 \mathrm{~K}-14986 \mathrm{~K}$ & $7.0-9.0$ & 0.0 & Sloan/UBVRI & LSM/Eq. (4) \\
\hline Table 18 & DA-3D & $5998 \mathrm{~K}-14986 \mathrm{~K}$ & $7.0-9.0$ & 0.0 & Sloan/UBVRI & LSM/Eq. (5) \\
\hline Table 19 & DA-3D & $5998 \mathrm{~K}-14986 \mathrm{~K}$ & $7.0-9.0$ & 0.0 & Sloan/UBVRI & LSM/Eq. (6) \\
\hline Table 20 & DA-3D & $5998 \mathrm{~K}-14986 \mathrm{~K}$ & $7.0-9.0$ & 0.0 & Sloan/UBVRI & LSM/Eq. (7) \\
\hline Table 21 & DA-3D & $5998 \mathrm{~K}-14986 \mathrm{~K}$ & $7.0-9.0$ & 0.0 & Sloan/UBVRI & LSM/Eq. (8) \\
\hline Table 22 & DA-3D & $5998 \mathrm{~K}-14986 \mathrm{~K}$ & $7.0-9.0$ & 0.0 & Sloan/UBVRI & LSM/Eq. (9) \\
\hline Table 23 & DB-3D & $12098 \mathrm{~K}-34105 \mathrm{~K}$ & $7.5-9.0$ & -10.0 & Kepler/TESS/Gaia/HiPERCAM & LSM/Eq. (4) \\
\hline Table 24 & DB-3D & $12098 \mathrm{~K}-34105 \mathrm{~K}$ & $7.5-9.0$ & -10.0 & Kepler/TESS/Gaia/HiPERCAM & LSM/Eq. (5) \\
\hline Table 25 & DB-3D & $12098 \mathrm{~K}-34105 \mathrm{~K}$ & $7.5-9.0$ & -10.0 & Kepler/TESS/Gaia/HiPERCAM & LSM/Eq. (6) \\
\hline Table 26 & DB-3D & $12098 \mathrm{~K}-34105 \mathrm{~K}$ & $7.5-9.0$ & -10.0 & Kepler/TESS/Gaia/HiPERCAM & LSM/Eq. (7) \\
\hline Table 27 & DB-3D & $12098 \mathrm{~K}-34105 \mathrm{~K}$ & $7.5-9.0$ & -10.0 & Kepler/TESS/Gaia/HiPERCAM & LSM/Eq. (8) \\
\hline Table 28 & DB-3D & $12098 \mathrm{~K}-34105 \mathrm{~K}$ & $7.5-9.0$ & -10.0 & Kepler/TESS/Gaia/HiPERCAM & LSM/Eq. (9) \\
\hline Table 29 & DB-3D & $12098 \mathrm{~K}-34105 \mathrm{~K}$ & $7.5-9.0$ & -10.0 & Sloan/UBVRI & LSM/Eq. (4) \\
\hline Table 30 & DB-3D & $12098 \mathrm{~K}-34105 \mathrm{~K}$ & $7.5-9.0$ & -10.0 & Sloan/UBVRI & LSM/Eq. (5) \\
\hline Table 31 & DB-3D & $12098 \mathrm{~K}-34105 \mathrm{~K}$ & $7.5-9.0$ & -10.0 & Sloan/UBVRI & LSM/Eq. (6) \\
\hline Table 32 & DB-3D & $12098 \mathrm{~K}-34105 \mathrm{~K}$ & $7.5-9.0$ & -10.0 & Sloan/UBVRI & LSM/Eq. (7) \\
\hline Table 33 & DB-3D & $12098 \mathrm{~K}-34105 \mathrm{~K}$ & $7.5-9.0$ & -10.0 & Sloan/UBVRI & LSM/Eq. (8) \\
\hline Table 34 & DB-3D & $12098 \mathrm{~K}-34105 \mathrm{~K}$ & $7.5-9.0$ & -10.0 & Sloan/UBVRI & LSM/Eq. (9) \\
\hline
\end{tabular}

